\title{
Structure and photoluminescence of helium-intercalated fullerite $\mathrm{C}_{60}$
}

\author{
I. V. Legchenkova, A. I. Prokhvatilov, Yu. E. Stetsenko, \\ M. A. Strzhemechny, K. A. Yagotintsev, A. A. Avdeenko, V. V. Eremenko, \\ P. V. Zinoviev, V. N. Zoryansky, and N. B. Silaeva \\ B. Verkin Institute for Low Temperature Physics and Engineering \\ of the National Academy of Sciences of Ukraine, 47 Lenin Ave., Kharkov 61103, Ukraine \\ E-mail: avdeenko@ilt.kharkov.ua
}

\section{R. S. Ruoff}

Northwestern University, Department of Mechanical Engineering 2145 Sheridan Road, Evanston, Illinois 60208, USA

Received September 25, 2002

\begin{abstract}
Intercalation of $\mathrm{C}_{60}$ single crystals with helium was studied by powder $\mathrm{x}$-ray diffractometry. It was established that the intercalation is a two-stage process, octahedral cavities are filled first and then tetrahedral ones, the chemical pressure being negative during both stages. For the first time low-temperature ( $5 \mathrm{~K}$ ) photoluminescence spectra of helium-intercalated fullerite $\mathrm{C}_{60}$ were studied. The presence of helium in lattice voids was shown to reduce that part of the luminescent intensity which is due to the emission of covalently bound pairs of $\mathrm{C}_{60}$ molecules, the so-called «deep traps» with the $0-0$ transition energy close to $1.69 \mathrm{eV}$. The mechanism of the effect of the intercalation with helium on the pair formation in fullerite $\mathrm{C}_{60}$ is discussed.
\end{abstract}

PACS: 61.48.+c, 61.66. $-\mathrm{f}, 71.35 . A a$, 78.55. $-\mathrm{m}$

The $\mathrm{C}_{60}$ fullerite has attracted attention during the last years owing to its unusual properties, such as unique optical and photoelectrical phenomena [1] and magnetism [2]. The high symmetry of the $\mathrm{C}_{60}$ molecule determines the geometric and electronic structure of its electronic excited states. Although $\mathrm{C}_{60}$ is a typical representative of $\pi$-electron systems and exhibit electronic properties inherent in these systems, the (quasi)degeneration of the lowest excited states and symmetry forbidden transitions between these states and the ground state, and the peculiarities of the low-temperature crystal structure are factors that contribute to the unusual low-temperature luminescence of fullerite $[3,4]$.

Despite the large number of publications about the low-temperature luminescence spectra of $\mathrm{C}_{60}$, the mechanisms that form these spectra are still far from clear. As it was recently shown, the low-tem- perature photoluminescence spectrum of the fullerite $\mathrm{C}_{60}$ is determined by a set of emission centers of different origin, such as Frenkel-Davydov excitons [3,4], charge transfer excitons [5], structural defects $[3,4,6,7]$, as well as pairs or chains of molecules that play the role of deep exciton traps, typical of the fullerites $\mathrm{C}_{60}$ and $\mathrm{C}_{70}$ [3,8-10].

In order to further investigate photoluminescence centers in the low-temperature phase of $\mathrm{C}_{60}$ we measured photoluminescence spectra of fullerite $\mathrm{C}_{60}$ saturated with helium (which has an effective atomic radius of $0.93 \AA$ ), which can presumably [11,12] bind to separate molecules in the crystal lattice of $\mathrm{C}_{60}$. In crystalline $\mathrm{C}_{60}$ there are two tetrahedral cavities and one octahedral cavity per fullerene molecule; the mean diameters of which are respectively 2.2 and $4.2 \AA[13,14]$. This allows the straightforward preparation of fullerite-based sub- 
stitutional solutions over a wide range of dopant concentrations. Surprisingly, given the ever growing number of publications in this field, no general review is yet available. For some types of impurities (for instance, alkali metals), there is a more or less generally accepted physical viewpoint concerning the effects brought about by the impurities, while the others (for instance, rare gases or simple linear molecules), such a unified concept is still to be worked out. Among the rare gas intercalants the effects of saturation of $\mathrm{C}_{60}$ with helium have been the least studied [11,12]. It is perhaps surprising that elevated pressures are not required to reach high helium concentrations in $\mathrm{C}_{60}$, though it is quite reasonable to expect that saturation kinetics as well as the consequences of intercalation will bear much similarity with results for other small-size intercalant species.

In the first stage of this work we studied the kinetics of saturation of fullerite $\mathrm{C}_{60}$ with $\mathrm{He}$ at room temperature and at pressures close to $10^{5} \mathrm{~Pa}$. For structure and luminescence investigations we used $\mathrm{C}_{60}$ single crystals from the same batch, the preparation procedure has been reported earlier [15]. Evolution of the intercalation process was monitored by means of powder x-ray diffraction (diffractometer DRON-3); the lattice parameters and other structure characteristics (reflection intensities and halfwidths) were measured at particular time intervals during a long lasting experiment at room temperature, with excursions to low (down to $20 \mathrm{~K}$ ) temperatures. A detailed description of these structure experiments will be reported in a separate publication. The conclusions about the distribution and saturation level drawn from the structure experiments were used for the interpretation of subsequent optical measurements.

The room-temperature cubic lattice parameter $a$ as a function of the exposure time of the uninterrupted intercalation process is plotted in Fig. 1. Noteworthy is the rather fast increase of $a$ during the initial 25-30 h of intercalation, which then converted to a much slower gradual increase with distinct indications of a tendency to saturate only after $4000 \mathrm{~h}$ of exposure to helium atmosphere. The half-width of all reflections has a maximum approximately in the same region where the saturation regime changeover was documented for the $a(t)$ dependence.

This temporal behavior of the structure characteristics allows us to tentatively reconstruct the scenario of helium penetration into the sample. During the first stage, octahedral cavities are filled, which are larger in size and have much wider passes in bet-

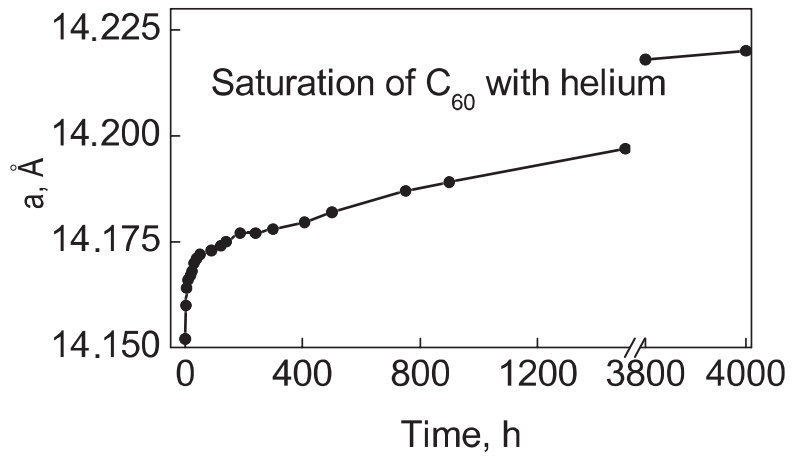

Fig. 1. Variations of the cubic lattice parameter $a$ of fullerite $\mathrm{C}_{60}$ versus the time of exposure to helium atmosphere at a pressure of around $10^{5} \mathrm{~Pa}$ and a temperature of $295 \mathrm{~K}$.

ween, as compared to tetrahedral cavities. A characteristic feature of the beginning of stage 1 is an inhomogeneous distribution of helium over the sample (helium diffuses from the sample surface inward), which manifests itself through a substantial broadening of the reflections. As the He concentration gets more homogeneous, the half-width is restored to a value that differs little from that in pure starting fullerite. After that, a much slower filling of tetrahedral vacancies begin. Estimates of the typical energies of helium in the respective environments of both vacancy types as well as the relevant diffusion problem will be published separately. It should be noted here that, unlike octahedral vacancies, the system of tetrahedral vacancies is filled virtually homogeneously over the whole sample. The He content can be roughly (to within 5-10\%) evaluated by comparing the Bragg reflection intensities calculated for known He fractions with measured intensities.

In the next stage of this work we studied effects of intercalation on low-temperature photoluminescence spectra of fullerite $\mathrm{C}_{60}$. The luminescence measurement technique, as well as the experimental setup have been reported elsewhere [7]. A minor modification consisted in using a FEU-62 $\left(\mathrm{S}_{1}\right)$ photomultiplier which enabled us to record photoluminescence spectra down to energies around $1.2 \mathrm{eV}$. Figure 2 shows photoluminescence spectra, normalized to integrated intensity, for pure fullerite and fullerite with helium impurities taken at $5 \mathrm{~K}$, as well as a difference between the two. This difference spectrum is consistent with the luminescence spectrum of «type $A$ » [4], which originates at the so-called deep traps (the $0-0$ transition energy being equal to $1.69 \mathrm{eV}$ ), formed by a pair or a chain of $\mathrm{C}_{60}$ molecules $[4,8,9]$. Thus, we can infer that the 


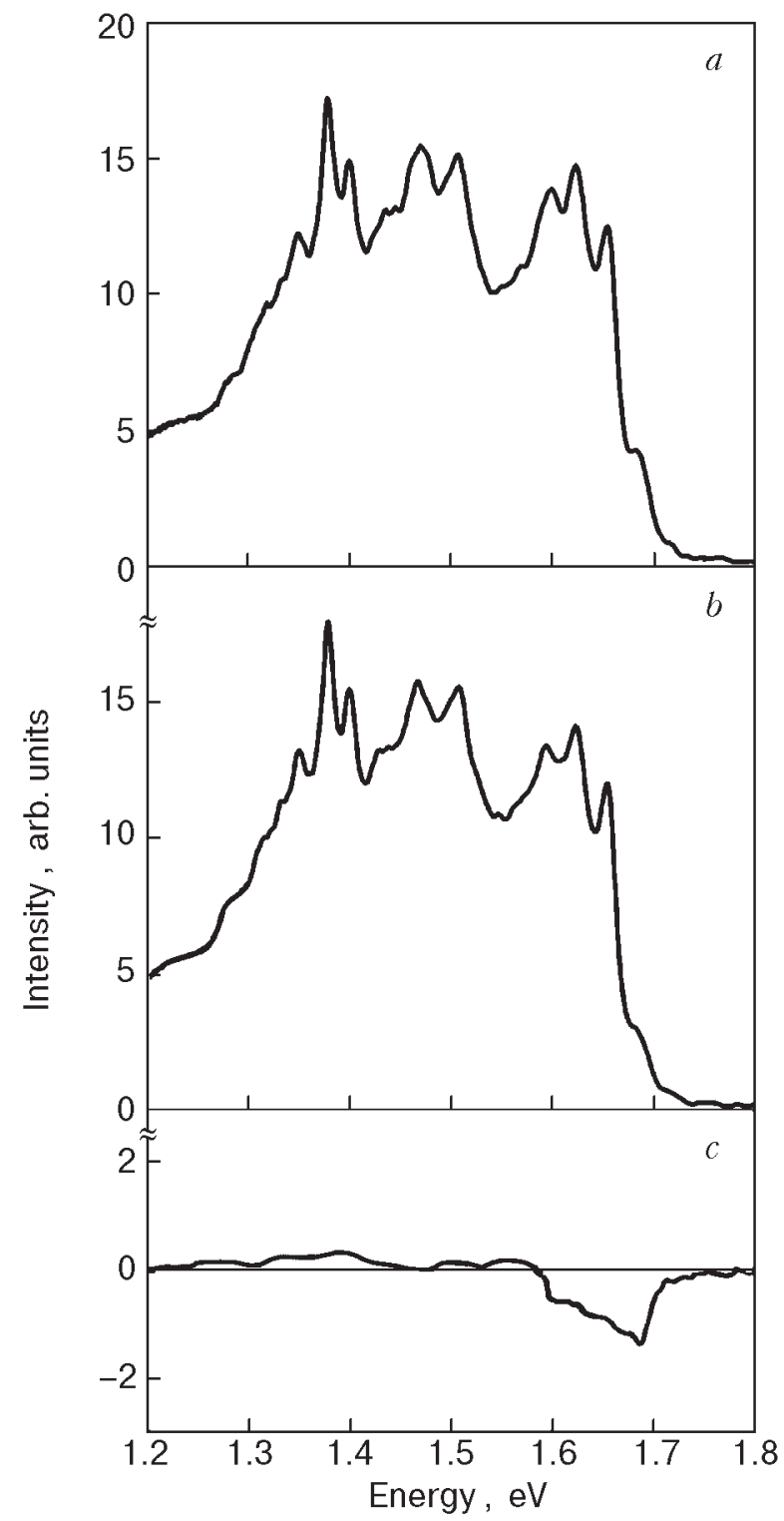

Fig. 2. Photoluminescence spectra of fullerite $\mathrm{C}_{60}$ at $5 \mathrm{~K}$ under excitation of light with energy $2.84 \mathrm{eV}$ : photoluminescence spectrum of pure $\mathrm{C}_{60}$, normalized to integrated intensity $(a)$; photoluminescence spectrum (normalized to integrated intensity) of $\mathrm{C}_{60}$ with helium impurities (time of exposure to helium at atmospheric pressure and room temperature is $440 \mathrm{~h}$ ) (b); difference spectrum obtained by subtracting the upper from lower ones $(c)$.

helium impurity, which can form a weak chemical bond with $\mathrm{C}_{60}$ and increase the crystal lattice parameter, hinders the formation of radiation centers that could be responsible for type- $A$ luminescence. A possible mechanism of this phenomenon might be as follows. As shown by Davydov et al. [16], polymerization of $\mathrm{C}_{60}$ in its low-temperature phase is affected by the number ratio of hexagon and pentagon pair-wise configurations, the hexagon configuration being the one that favors polymerization. The helium impurity should promote a depletion of hexagon configurations due to larger lattice parameters in doped crystals and, thereby, inhibit polymerization, which is often initiated by stresses around dislocations [9]. The only known mechanism of producing covalent bonding in the low-temperature phase of $\mathrm{C}_{60}$ is polymerization in distorted areas around dislocations [9,16]. Generally speaking, since dimerization is hardly possible even in a purely hexagonal state of $\mathrm{C}_{60}$, orientational disorder, irrespective of its origin, is the necessary condition for the formation of covalent dimers. The presence of orientation disorder in the $\mathrm{C}_{60}$ low-temperature phase is confirmed by the inhomogeneous broadening of the photoluminescence bands, which is removed using site selective excitation [4]. Thus, the hypothesis that the type- $A$ luminescence spectrum in the low-temperature phase of fullerite $\mathrm{C}_{60}$ is due to covalent bonded molecules, is confirmed by our measurements on $\mathrm{C}_{60}$ intercalated with helium.

In conclusion, based on x-ray measurements we conclude that ambient-pressure room-temperature intercalation of $\mathrm{C}_{60}$ with helium is accomplished in two stages. First, the octahedral voids are comparatively quickly filled, while during the second stage a much slower filling of tetrahedral vacancies occurs. In this paper we show for the first time that intercalation of $\mathrm{C}_{60}$ fullerite with helium gas leads to an intercalated material that displays an appreciably reduced type- $A$ luminescence in the low-temperature phase. This effect is tentatively ascribed to the hindrance of polymerization owing to a decrease in hexagon configuration content in helium-intercalated fullerite $\mathrm{C}_{60}$.

1. Optical and Electronic Properties of Fullerenes and Fullerene Based Materials, J. Shinar, Z. V. Vardeny, and Z. H. Kafafi (eds.), Marcel Dekker, New York - Basel (2000).

2. T. L. Makarova, B. Sundqvist, R. Höhne, P. Esquinazi, Y. Kopelevich, P. Sharff, V. A. Davydov, L. S. Kashevarova, and A. V. Rakhmanina, Nature 413, 716 (2001).

3. V. Capozzi, M. Santoro, G. Perna, G. Celentano, A. Minafra, and G. Casamassima, Eur. Phys. J. Appl. Phys. 14, 3 (2001).

4. I. Akimoto and K. Kan'no, J. Phys. Soc. Jpn. 71, 630 (2002).

5. S. Kazaoui, N. Minami, Y. Tanabe, H. J. Byrne, A. Eilmes, and P. Petelenz, Phys. Rev. B58, 7689 (1998).

6. W. Guss, J. Feldmann, E. O. Göbel, C. Taliani, H. Mohn, W. Müller, P. Häussler, and H.-U. ter Meer, Phys. Rev. Lett. 72, 2644 (1994). 
7. A. A. Avdeenko, N. I. Gorbenko, V. V. Eremenko, P. V. Zinoviev, A. T. Pugachev, N. B. Silaeva, Yu. A. Tiunov, and N. P. Churakova, Fiz. Nizk. Temp. 25, 49 (1999) [Low Temp. Phys. 25, 37 (1999)].

8. D. J. van den Heuvel, G. J. B. van den Berg, E. J. J. Groenen, J. Schmidt, I. Holleman, and G. Meijer, J. Phys. Chem. 99, 11644 (1995).

9. D. V. Dyachenko-Dekov, Yu. V. Iunin, A. N. Izotov, V. V. Kveder, R. K. Nikolaev, V. I. Orlov, Yu. A. Ossipyan, N. S. Sidorov, and E. A. Steinman, Phys. Status Solidi B222, 111 (2000).

10. M. Ichida, S. Tanaka, and A. Nakamura, J. Lumin. 87-89, 785 (2000).

11. K. Ichimura, K. Imaeda, and H. Inokuchi, Chem. Lett. 196 (2000).

12. K. Ichimura, K. Imaeda, and H. Inokuchi, Mol. Cryst. Liq. Cryst. 340, 649 (2000).
13. P. A. Heiney, J. Phys. Chem. Solids 53, 1333 (1992).

14. C. H. Pennington and V. A. Stenger, Rev. Mod. Phys. 68, 855 (1996).

15. L. S. Fomenko, V. D. Natsik, S. V. Lubenets, V. G. Lirtsman, N. A. Aksenova, A. P. Isakina, A. I. Prokhvatilov, M. A. Strzhemechny, and R. S. Ruoff, in: Recent Advances in Chemistry and Physics of Fullerenes, K. Kadish and R. Ruoff (eds.), v. 2, Electrochemical Soc., Pennington (1995), p. 926.

16. V. A. Davydov, L. S. Kashevarova, A. V. Rakhmanina, V. M. Senyavin, O. P. Pronina, N. N. Oleynikov, V. Agafonov, R. Ceolin, H. Allouchi, and H. Szwarc, Chem. Phys. Lett. 333, 224 (2001). 